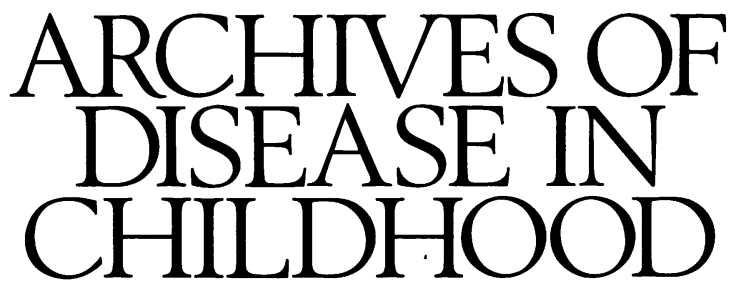

The fournal of the British Paediatric Association

\title{
Annotations
}

\section{Use of antiepileptic drugs in childhood epilepsy}

Attitudes to the treatment of childhood epilepsy with antiepileptic drugs have been changing during the past decade. It is now widely accepted that epilepsy in children is very different from epilepsy in adults and that studies of drug treatment in adults are not necessarily directly applicable to children. ${ }^{1}$ Before starting treatment with these drugs in a child it is important to establish a firm diagnosis of epilepsy, to identify the type of epilepsy or epileptic syndrome which is present, as far as is feasible, and to categorise the seizure type or types in the individual patient.

The epilepsies or epileptic syndromes may broadly be divided into those with no established aetiology but which are probably of genetic origin (idiopathic or primary) and those with a known aetiology (symptomatic or secondary) in which a brain lesion is suspected or can be demonstrated. A further subdivision is into those epilepsies with generalised seizures (generalised epilepsies) and those with partial seizures (partial or localisation related epilepsies). ${ }^{2}$ It should be remembered, however, that similar seizures may occur in different syndromes and that some syndromes are characterised by multiple seizure types. While some epileptic syndromes are highly specific and a few have recently been shown to have chromosomal markers, many represent broad concepts only. ${ }^{3}$ Furthermore, not all patients fit into the described syndromes. Nevertheless, this approach to childhood epilepsy had undoubtedly improved the management of the condition in recent years and has led to more rational use of the available drugs.

The drug treatment of epilepsy always needs to be considered on a risk versus benefit basis as no drug is free of risk, although modern drugs are superior in this respect especially where cognition and behaviour are concerned. ${ }^{4}$ Furthermore, the need to treat at all should be debated as, in many of the primary epilepsies, attacks are infrequent anyway and the outcome is usually excellent. ${ }^{56}$ Two common disorders of this kind are benign partial epilepsy with rolandic (centrotemporal) spikes and febrile convulsions. In the former, up to one quarter of the patients have one seizure only with one half having fewer than five seizures. ${ }^{7}$ In the case of febrile convulsions, the therapeutic pendulum has swung strongly against pharmacological prophylaxis during the past decade, to be replaced by intermittent forms of treatment using diazepam rectally. ${ }^{8}$ Single seizures are also a feature of some of the primary generalised epilepsies, notably when an idiopathic tonic-clonic seizure occurs in an otherwise normal child with a normal electroencephalogram. In such a situation, recurrences may not be seen in over half of the cases. ${ }^{9} 10$ However,the observations of Reynolds on untreated epilepsy should be borne in mind. ${ }^{11}$ His collaborative study has demonstrated, in children as in adults, a significant and important relationship between the number of seizures before treatment and the ultimate prognosis. ${ }^{12}$ When a second attack follows quickly on the first (in a month or less), early institution of treatment is mandatory and may influence the long term outcome.

If drug treatment of epilepsy is started, it should be withdrawn as early as is feasible. In children, remission rates of over $75 \%$ have been consistently reported in those who have been seizure free for two years. ${ }^{13} 14$ The benign nature of much of childhood epilepsy needs to be more widely recognised. The long term prognosis for children with epilepsy is nearly always closely related to the type of epileptic syndrome which is present and ultimately to the aetiology. The presence of structural brain abnormality, mental and developmental handicaps, and certain seizure types are associated with a worse prognosis.

With refinements in diagnosis and with the availability of accurate monitoring of drug concentrations has come the realisation that the treatment of epilepsy with a single drug (monotherapy) is superior to the use of multiple drug treatment. It is now clear that polytherapy was responsible for much unnecessary and avoidable drug toxicity in the past. It also led to ineffective treatment because of drug interactions and to difficulties in evaluating the effects of individual drugs. Another feature of multiple drug treatment is a poor risk-benefit ratio that is revealed when the efficacy of drug combinations is critically assessed. ${ }^{15}$ In the treatment of newly diagnosed patients with epilepsy, whether adults or children, the evidence now strongly supports the value of antiepileptic drug monotherapy and approximately $75 \%$ of patients can be expected to enter prolonged remissions on such a regime. ${ }^{12}$ If the drug chosen initially proves ineffective at the highest tolerated dosage, a second drug should be tried alone. Drug combinations may be justified in patients with resistant disease, although such combinations are rarely successful. An exception is the combination of ethosuximide and sodium valproate for resistant childhood absence epilepsy (petit mal). ${ }^{16}$ Adjunctive or 'add-on' treatment with drugs such as clobazam or acetazolamide may sometimes be effective in controlling intractable seizures in certain epileptic syndromes. ${ }^{17}$

Because young children tend to metabolise antiepileptic drugs more rapidly than older individuals, they may require 
higher dosage in relation to body weight. ${ }^{1}$ Determining blood concentrations of drugs is important in children but it should be remembered that the so called 'therapeutic range' is a relative concept and should not be applied blindly. ${ }^{18}$ As much drug as is needed but as little as is necessary to control the seizures should be given, ${ }^{6}$ polytherapy should be avoided, and drug concentration monitoring used widely. An added inducement to use low doses where possible is that, in patients on monotherapy with various drugs, the effects on memory, attention and concentration, perception and decision making, are twice as great at a high serum concentration as compared with a low serum concentration in the same patient. ${ }^{19}$

Although factors such as seizure type and the underlying aetiology of the epilepsy influence prognosis, the influence of choice of antiepileptic drug on the prognosis is less clear. Leaving aside childhood absence epilepsy where ethosuximide and/or sodium valproate are uniquely effective, controlled studies to determine the relative efficacy of four major antiepileptic drugs, that is, phenobarbitone, phenytoin, carbamazepine, and sodium valproate, utilised as monotherapy, showed no significant differences in efficacy in either adults or children, regardless of which method of patient assessment was employed. ${ }^{12}$ However, bearing in mind the known effects on cognition and behaviour of the barbiturates (including primidone), phenytoin and the benzodiazepines, ${ }^{19}$ paediatricians will opt to use carbamazepine and sodium valproate as their drugs of first choice. Although carbamazepine probably has only a minimal adverse effect in these respects, sodium valproate may affect behaviour, apparently as an idiosyncratic effect, and can also impair cognition. ${ }^{20}$

A more worrying problem with sodium valproate is the possibility of fatal hepatotoxicity developing as an idiosyncratic reaction, usually within the first 90 days of treatment. It should be remembered that, although drug related rises in serum enzyme activities may be detected in up to $44 \%$ of patients receiving valproate treatment, ${ }^{21}$ these rises are not accompanied by clinical symptoms or by abnormalities in the liver's synthetic functions (for example, prothrombin time). These laboratory abnormalities resolve with dosage reduction or with stopping the drug. The incidence of fatal hepatotoxicity is not evenly distributed among the patient population. Very young patients $(0-2$ years) on polytherapy with antiepileptic drugs and who have other medical conditions in addition to epilepsy are disproportionately vulnerable to fatal hepatic failure. ${ }^{22}$ This is usually attributed to an aberration in the metabolism of the drug and may, in some cases, be associated with an inborn metabolic defect. ${ }^{23}$ Sodium valproate prophylaxis for febrile convulsions is, therefore, not really an option under 2 years of age, except in exceptional circumstances, and short term phenobarbitone treatment is a wiser choice in the rare instances when continuous treatment is deemed necessary. ${ }^{8}$

The good news about sodium valproate treatment is that the incidence of fatal hepatotoxicity from the drug is declining. ${ }^{24}$ This has been ascribed to the increasing use of the drug as monotherapy and the limitation of its use to low risk patients (over 2 years). Sodium valproate should not be given in a polytherapy regime to those under 3 years of age, it should be avoided in patients with pre-existing liver disease or with a family history of such disease, and salicylates should not be given to a patient on sodium valproate. Fasting should be avoided in slich patients who have an intercurrent illness, and immediate withdrawal of the drug is mandatory if hepatotoxicity is suspected. Prodromal signs of this are nausea, vomiting, anorexia, lethargy, and also a sudden loss of seizure control or even an episode of status epilepticus. ${ }^{25}$ One stage prothrombin time and fibrinogen concentrations are more sensitive than other indices of liver function in detecting hepatic compromise. Although valproate hepatotoxicity is rare, it may occur at any age and vigilance is required to detect its early onset.

Despite these caveats about sodium valproate, both it and carbamazepine are widely used today in the treatment of childhood epilepsy. It is worth entering a caveat here about carbamazepine also which, apart from its annoying propensity to cause a generalised rash just when its anticonvulsant properties are most needed, may also exacerbate seizures, especially when used to treat the multiple seizure types of the Lennox-Gastaut syndrome or absence scizures of various kinds. ${ }^{26}$ Carbamazepine, in the writer's experience, has been an outstanding and safe antiepileptic drug for treating childhood epilepsy over nearly three decades. It is particularly effective in simple and complex partial seizures. Sodium valproate is usually very effective in generalised convulsive and non-convulsive seizures, especially when they are due to a primary or idiopathic epileptic syndrome and are associated with $3 \mathrm{~Hz}$ spike wave discharges on electroencephalography.

These are several new antiepileptic drugs in the course of development ${ }^{27}$ and two have arrived recently on the commercial market. Both of these have been developed as a result of a logical pharmacological approach to treatment, which has attempted to find drugs which either enhance synaptic inhibition or reduce excitatory neurotransmission. The former is largely mediated by $\gamma$-aminobutyric acid (GABA) and $\gamma$-vinyl- $\gamma$-aminobutyric acid (vigabatrin) is a synthetic derivative of GABA which acts as an irreversible inhibitor of GABA transaminase, the enzyme responsible for the breakdown of GABA. Its action results in an increase in inhibitory neurotransmitter at the synapse. The drug is not bound to protein, is excreted largely unchanged in the urine, and monitoring of serum concentrations is not advised. Vigabatrin has been used so far as adjunctive treatment for chronic and intractable seizures in adults and, in these, about half of the patients treated have shown a reduction in seizure frequency. ${ }^{28}$ Partial seizures with secondary generalisation have shown the best response. Thus far, experience in children is limited and confined to intractable cases but uncontrolled partial seizures seem to respond best and myoclonic seizures may also show a response, especially the intractable seizures of West's syndrome (infantile spasms) due to tuberous sclerosis. ${ }^{29}$ Although vacuolar changes in myelin were reported in some animals given vigabatrin, clinical effects resulting from something of that nature have never been reported in man. Nevertheless, vigilance for evidence of chronic toxicity is required, especially in children. The drug will clearly have an important role in the treatment of resistant epilepsy in the future and may begin to be used more widely as first line rather than adjunctive treatment.

The other new antiepileptic drug, lamotrigine, is designed to act by inhibiting the release of excitatory amino acids, principally glutamate, at the synapse. It is recommended for use at present as adjunctive treatment for partial seizures and generalised tonic-clonic seizures that are not satisfactorily controlled by other antiepileptic drugs. ${ }^{30}$ These older drugs may increase its metabolism by liver enzyme induction, although sodium valproate reduces it. It seems to be relatively non-toxic although rashes may occur. Experience in childhood is limited and a multicentre trial of the drug as adjunctive treatment for resistant epilepsy is still in progress in the UK.

As was emphasised earlier, epilepsy in childhood is quite a different problem from epilepsy in adults and its treatment with or without drugs is influenced by many factors unique to that period of life. The British Paediatric Association, in 1988, emphasised the importance of better control of epilepsy among the priorities for child health in the future. ${ }^{31}$ 
Interest in and understanding of epilepsy have never been greater than at present and, with the rapid development of knowledge of the molecular biology and neurochemical mechanisms underlying epilepsy, it is likely that research will provide more effective and safer drugs in the near future.

N V O'DONOHOE

Department of Paediatrics,

(Trinity College),

National Children's Hospital,

Harcourt Street,

Dublin 2,

Republic of Ireland

1 Dodson WE. Special pharmacokinetic considerations in children. Epilepsia 1987;28 (suppl 1):S56-70.

2 Commission on Classification and Terminology of the International League Against Epilepsy. Proposal for revised classification of epilepsies and epileptic syndromes. Epilepsia 1989;30:389-99.

3 O'Donohoe NV. The epilepsies. In: Eyre J, Boyd R, eds. Paediatric specialty practice for the 1990s. London: Royal College of Physicians, 1991:51-63.

4 Abu-Arafeh IA, Wallace SJ. Unwanted effects of antiepileptic drugs. Dev Med Child Neurol 1988;30:117-20.

5 Taylor DC, McKinlay I. When not to treat epilepsy with drugs. Dev Med Child Neurol 1984;26:822-33.

6 Freeman JM. A clinical approach to the child with seizures and epilepsy. Epilepsia 1987;28(suppl 1):S103-7.

7 Beaussart M, Faou R. Evolution of epilepsy with rolandic paroxysmal foci. A study of 324 cases. Epilepsia 1978;19:333-42.

8 Addy DP. Phenobarbitone and febrile convulsions. Arch Dis Child 1990;65: 921.

9 Hirtz DG, Ellenberg JH, Nelson KB. The risk of recurrence of non-febrile seizures in children. Neurology 1984;34:637-41.

10 Camfield P, Camfield C, Dooley J, Tibbles J, Fung T, Garner B. Epilepsy after a first unprovoked seizure in childhood. Neurology 1985;35:1657-60.

11 Reynolds EH. The process of kindling: is kindling relevant? In: Bolwig TG Trimble MR, eds. The clinical relevance of kindling. Chichester: John Wiley
1989:149-60.

12 de Silva M, McArdle B, McGowan M, Reynolds EH, Neville B, Johnson AL. Monotherapy for newly diagnosed childhood epilepsy. A comparative tria and prognostic evaluation. Epilepsia 1989;30:662.

13 Shinnar S, Vining EPG, Mellits EM, et al. Discontinuing antiepileptic medication in children with epilepsy after two years without seizures. $N E n g l$ cation in children with

14 Matricardi M, Brinciotti $\dot{M}$, Benedetti P. Outcome of discontinuation of antiepileptic drug treatment in children with epilepsy. Epilepsia 1989;30:582-9. epileptic drug treatment in children with epilepsy. Epilepsia 1989;30:582-9. Schmidt D. Adverse effects of anti-ep
$\mathcal{F}$ Med 1989;56(suppl, part 1):S132-9.

16 Rowan AJ, Meijer JWA, De-Beer Pawlikowski N, Van Der Geest P, Meinardi $\mathrm{H}$. Valproate-ethosuximide combination therapy for refractory absence seizures. Arch Neurol 1983;40:797-802.

17 Callaghan N, Goggin T. Ádjunctive therapy in resistant epilepsy. Epilepsia 1988;29(suppl 1):S29-35.

18 Wallace SJ. Antiepileptic drug monitoring. An overview. Dev Med Child Neurol 1990;32:923-6.

19 Trimble MR. Anticonvulsant drugs: mood and cognitive function. In: Trimble MR, Reynolds EH, eds. Epilepsy, behaviour and cognitive function. Chichester: John Wiley, 1988: 135-43.

20 Gallasi R, Mooreale A, Lorusso S, Rocaccianti G, Lugaresi E, Baruzzi A. Cognitive effects of valproate. Epilepsy Res 1990;5:160-4.

21 Sussman NM, McLain LW. A direct hepatotoxic effect of valproic acid. $7 A M A$ 1979;242:1173-4.

22 Brown JK. Valproate toxicity. Dev Med Child Neurol 1988;30:121-5.

23 Appleton RE, Farrell K, Applegarth DA, Dimmick JE, Wong LTK, Davidson AGF. The high incidence of valproate hepatotoxicity in infants may relate to familial metabolic defects. Can f Neurol Sci 1990;17:145-8.

24 Dreifuss FE, Langer DH, Moline KA, Maxwell JE. Valproic acid hepatic fatalities. II. US experience since 1984. Neurology 1989;39:201-7.

25 Dreifuss FE, Langer DH. Hepatic considerations in the use of antiepileptic drugs. Epilepsia 1987;28(suppl 2):S23-9.

26. Snead OC, Hosey LC. Exacerbation of seizures in children by carbamazepine. N Engl Y Med 1985;313:916-21.

27 Brodie MJ, Porter RJ. New and potential anticonvulsants. Lancet 1990;336: 425-6.

28 Reynolds EH. Vigabatrin. Rational treatment for chronic epilepsy. BMF 1990;300:277-8.

29 Luna D, Dulac O, Pajot N, Beaumont D. Vigabatrin in the treatment of childhood epilepsy. A single-blind placebo-controlled study. Epilepsia 1989;30:430-7.

30 Jawad S, Richens A, Goodwin G, Yuen WC. Controlled trial of lamotrigine (Lamictal) for refractory partial seizures. Epilepsia 1989;30:356-63.

31 Court D, Alberman E. Worlds apart. In: Forfar JO, ed. Child health in a changing society. Oxford: Oxford University Press foi the British Paediatric Association, 1988:1-30.

\section{Transoesophageal echocardiography: a new diagnostic method in paediatric cardiology}

Over the past decade cardiac ultrasound techniques have revolutionised the practice of paediatric cardiology. Transthoracic cross sectional imaging allows for an accurate and non-invasive assessment of intracardiac morphology in the vast majority of children. Doppler ultrasound techniques provide a detailed insight into the related haemodynamic changes of congenital cardiac lesions.

Transoesophageal echocardiography has become an established diagnostic and monitoring technique in adult cardiology. ${ }^{1}$ An ultrasound transducer, mounted at the tip of a flexible endoscope, is introduced into the oesophagus and echocardiographic studies can be performed without interference of either the thoracic cage or lung tissue. This produces high quality images in every patient studied. Some two years ago, miniaturised transoesophageal probes were developed, ${ }^{2}$ which allow studies to be carried out in even small children. ${ }^{34}$ However, what are the advantages and what are the relative contributions of transoesophageal studies in children with congenital heart disease? Do we need a further diagnostic and monitoring technique?

Over a two year period more than 240 transoesophageal studies have been performed at the Sophia Childrens Hospital, Rotterdam, the Netherlands and the Royal Hospital for Sick Children, Edinburgh, Scotland. The results of these studies were reviewed to determine the role of paediatric transoesophageal studies in different clinical settings. All transoesophageal studies were carried out under either general anaesthesia (given in the majority of cases for either cardiac catheterisation or cardiac surgery) or heavy sedation. Antibiotic endocarditis prophylaxis was not administered in any patient, and no episode of bacterial endocarditis was observed. Complications were encountered in three children (1.2\%). These included arrhythmias in two. Oesophageal bleeding was encountered in only one patient. No death occurred.

\section{Primary diagnosis of congenital heart disease}

When compared with transthoracic echocardographic studies, the transoesophageal ultrasound approach provides a more detailed evaluation of the morphology of the atrial chambers. The visualisation of both atrial appendages, for the first time, allows the direct diagnosis of atrial situs ${ }^{5}$ and the definite preoperative documentation of juxtaposed atrial appendages. ${ }^{6}$ The morphology and integrity of the atrial septum is frequently better assessed by transoesophageal studies. Superiorly located defects, such as sinus venosus atrial septal defects, can be visualised in much greater 